\title{
Macroscopic White Blood Cell Casts: An Extremely Rare Presentation of Klebsiella Pyelonephritis
}

\author{
Larry B. Mellick, MD;; Kimberly M. Rathbun, MD \\ Department of Emergency Medicine, University of South Alabama, USA
}

\section{"Corresponding author}

Larry B. Mellick, MD

Professor, Division Chief, Department of Emergency Medicine, University of South Alabama, USA;Tel. 706-533-293 I;

E-mail: Imellick@health.southalabama.edu

\section{Article information}

Received: November 3 ${ }^{\text {rd }}, 2020$; Revised: December 10 $0^{\text {th }}$ 2020; Accepted: December 17 th $^{\text {th }} 2020$; Published: December $21^{\text {st }}, 2020$

\section{Cite this article}

Mellick LB, Rathbun KM. Macroscopic white blood cell casts:An extremely rare presentation of Klebsiella pyelonephritis. Emerg Med Open J. 2020; 6(I): 9-I0.

doi: 10.17|40/EMOJ-6-157

\section{ABSTRACT}

In this case report, we present a patient with severe pyelonephritis who presented complaining of passing worm-like tissue from his penis. This patient was passing macroscopic ureteral white blood cell casts resulting from a severe pyelonephritis caused by Klebsiella pneumoniae.

Keywords

Acute pyelonephritis; White blood cell casts; Urinary tract infections; Urinary tract infection (UTI); Ureteral casts.
}

\section{OVERVIEW}

W hite blood cell casts are extremely rare and only one previous report was found in our non-systematic review of the literature.

\section{CASE REPORT}

A 33-year-old previously healthy man presented to the emergency department with a one-week history of painful urination, body aches, fatigue, nausea, vomiting, and chills. On the morning of presentation, he reported passing worm-like "tissue" that "shot out" of his penis following an intense urge to urinate (Figure 1). At presentation, he was afebrile, and his vital signs were within normal limits. His physical examination was remarkable only for tenderness to palpation of the left lower abdominal quadrant and left flank. Urinalysis, urine culture, blood cultures, complete blood count and a computerized tomography (CT) of the abdomen and pelvis were ordered. The specimen was sent for culture and pathologic examination.

Laboratory testing demonstrated a leukocytosis of 20,600 white blood cells $/ \mathrm{mm}^{3}$. The urinalysis was nitrite negative, the leukocyte esterase was reported as "moderate" and the microscopic examination demonstrated too numerous to count white blood cells. The abdominal CT scan documented a severe le$\mathrm{ft}$ pyelonephritis with early intrarenal abscess formation and a suspected ureteral cast protruding into the bladder. The patient's u-

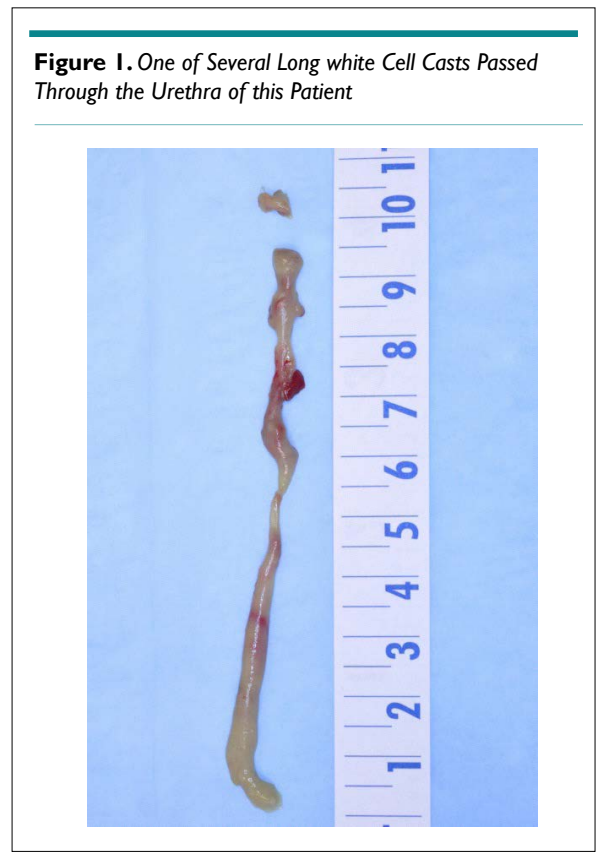


rine culture grew Gram-negative lactose-fermenting bacilli, and one of two blood cultures was positive for Klebsiella pneumoniae. A portion of the tissue specimen was sent for culture and grew Klebsiella pneumoniae. The pathology report described "mostly degenerated fibrinopurulent debris with abundant granulocytes, lymphocytes, and histiocytes". It also reported "some filamentous organisms and amorphous, refractile material present". The final pathology assessment was that the material most likely represented a tubular cast formed in the patient's ureter by abundant purulent material secondary to his pyelonephritis. The patient was treated with piperacillin and tazobactam and made a full recovery. Neither the CT scan on admission nor a second CT scan several days later demonstrated any anatomical variations or abnormalities.

\section{DISCUSSION}

This case describes a macroscopic ureteral white blood cell cast resulting from a severe pyelonephritis. Urinary tract infections and pyelonephritis, a complicated urinary tract infection, are common maladies treated in the emergency department. A population-based study of acute pyelonephritis in the United States found overall annual pyelonephritis rates (out patient) of 10-13 cases per 10,000 females and 2-3 cases per 10,000 males. ${ }^{1}$ While it is common to find microscopic white blood cell casts in the urine of patients diagnosed with pyelonephritis, ${ }^{2}$ macroscopic white blood cell casts that form of the ureter in association with pyelonephritis appear to be extremely rare. This is the first report of a macroscopic ureteral white blood cell cast since 1901 when it was first described in the British Medical Journal. $^{3}$

The most frequent cause of acute complicated urinary tract infectionsis Escherichia coli. Other uropathogens causing pyelonephritis are Enterobacteriaceae (such as Klebsiella spp. and Proteus spp.), Pseudomonas, enterococci, and staphylococci (methicillin-sensitive Staphylococcus aureus [MSSA] and methicillinresistant $S$. Aureus [MRSA]). ${ }^{1}$ Our patient was previously healthy and had no other known comorbidities or risk factors for a complicated pyelonephritis leading to the more severe disease presentation.

\section{CONCLUSION}

While renal corticomedullary abscess, perinephric abscess, emphysematous pyelonephritis, and papillary necrosis are recognized complications of pyelonephritis, the development of large, worm-like white cell casts is extremely rare. In fact, our patient appears to be only the second case of white blood cell casts reported in the literature.

\section{CONSENT}

No consent was required for this deidentified case report and single case reports are exempt from institutional review board (IRB) review.

\section{REFERENCES}

1. Czaja CA, Scholes D, Hooton TM, Stamm WE. Populationbased epidemiologic analysis of acute pyelonephritis. Clin Infect Dis. 2007; 45(3): 273-280. doi: 10.1086/519268

2. Ringsrud KM. Casts in the urine sediment. Lab Med. 2001; 32(4): 191-193. doi: 10.1309/KJ5E-V5FE-MAHR-KXT2

3. White JA. A case of renal colic attended by the passage of casts of the ureter (Ureteritis Membranacea). Br Med J. 1901; 1(2088): 14-15. doi: 10.1136/bmj.1.2088.14-a 